\title{
La infancia en riesgo social desde la sociedad del bienestar.
}

\author{
M. Ángeles Balsells \\ balsellsepip.udl.es \\ Universidad de Lleida
}

\section{Introducción}

El constante desarrollo de las sociedades avanzadas hace necesario un esfuerzo permanente de reconceptualización de aquellos fenómenos que se dan en su seno; no podemos eludir el compromiso científico de estar atentos a los nuevos fenómenos sociales y las consecuencias que tienen en el desarrollo individual del sujeto y en su educación. La definición de colectivos que por sus características propias y contextuales se encuentran en situaciones de riesgo social es una de las que más afectada se ve por estos fenómenos de cambio social, ya que los cambios estructurales, socioeconómicos y culturales son los que definen sus procesos de socialización delimitando, a su vez, las dificultades que se pueden encontrar,

Concretamente, dentro del vertiginoso dinamismo de las sociedades avanzadas, se están dando importantes cambios y apareciendo situaciones hasta ahora desconocidas, que afectan directamente a la correcta socialización de nuestra infancia. El colectivo que se recoge bajo el epígrafe de infancia en situación de riesgo se suele definir como un grupo en proceso de desadaptación social por causas básicamente familiares. Si hacemos un rápido repaso a las diferentes orientaciones teóricas que definen las situaciones de riesgo social veremos como el énfasis siempre recae en las circunstancias familiares.

Así, el enfoque socio-ambientalista acentúa la importancia de los factores ambientales y sus programas de intervención hacen hincapié en los apoyos a las familias cuando analiza este fenómeno. El enfoque conductista, en cambio, se centra en la idea que las situaciones de riesgo están causadas, fundamentalmente por las dificultades de los padres en desarrollar su rol parental y en cubrir las necesidades básicas de sus hijos; por otro lado las perspectivas psico-dinámicas ven la conducta como algo sintomático causado por experiencias pasadas que mucho tienen que ver con las habilidades afectivas familiares. Por último, el enfoque sistémico parte de la premisa de que el individuo con problemas forma parte de un sistema, básicamente familiar, con dificultades (Guasch, M. y Ponce, C. , 2002).

Por lo tanto, tal y como se observa, los enfoques teóricos que abordan la epistemología de la infancia en situación de riesgo se centran en la situación familiar, identificando las causas y los factores de riesgo en el microsistema familiar: la desestructura familiar, los maltratos infantiles familiares, la dinámica conflictiva entre padres e hijos, la ausencia o el exceso de disciplina, la negligencia o la ausencia de lazos afectivos,... son algunos de los factores que se han identificado como causa de la desadaptación social.

Lógicamente, la fuerza socializadora de la familia es tal, que no se puede negar que todos estos factores tienen una influencia directa sobre los procesos de desajuste entre el sujeto y la sociedad. Sin embargo, las preguntas a plantear son, ¿solo la familia puede reunir indicadores que explican las situaciones de riesgo?, ¿existen otras situaciones, fuera de la familia, que provocan la aparición de poblaciones infantiles en situación de riesgo social? ¿se puede hablar de infancia en situación de riesgo social cuando no existen maltratos o negligencia en la familia? En definitiva, ¿las sociedades 
desarrolladas y avanzadas son el escenario de nuevos grupos de riesgo social?

\section{Hacia una reconceptualización de la infancia en situación de riesgo social}

Efectivamente, las sociedades avanzadas han generado nuevos escenarios y nuevas problemáticas en la socialización de la infancia, provocando una multiplicidad de factores que pueden hacer aparecer de grupos de riesgo hasta ahora inexistentes. La reconceptualización del concepto, por lo tanto, debe pasar por la amplitud de mira hacia esas nuevas problemáticas sociales que provocan que niños y niñas que crecen en entornos familiares normalizados puedan verse inmersos en procesos de desadaptación o exclusión social por otras causas. Veamos cuales podrían ser los tres parámetros básicos que nos pueden hacer llegar a una definición más amplia.

En primer lugar, cabe destacar la importancia del medio en cualquier proceso de riesgo social y de desadaptación; para definir esta población cabe describir las situaciones carenciales que suelen caracterizar la familia y el entorno social inmediato de los niños. Un niño está en riesgo social si pertenece a un entorno que presenta factores de riesgo. En el reciente informe sobre "La infancia i les famílies als inicis dels segle XXI", se plantea estudiar el riesgo social a través de indicadores situados en la familia, en el sujeto y en sus entornos. (Gomez-Granell, Garcia-Mila, Ripol-Millet y Panchon, 2002).

En segundo lugar, también destacar la importancia de la interacción del niño con ese medio; la situación carencial entra en relación con las características personales del niño, de tal manera que se van generando respuestas activas en él y consolidando consecuencias en su socialización y sus características personales, cognitivas y afectivas. En tercer lugar, la consideración de la situación de la infancia en riesgo como una parte de un proceso hacia situaciones de inadaptación social.

Así, podemos considerar que la infancia en situación de riesgo social es aquella que establece, de forma procesal y dinámica, una interacción ideográfica e inadecuada con sus entornos, los cuales no cubren sus derechos inalienables, poniendo en peligro su correcto desarrollo y dando lugar a un posible inicio del procesos de inadaptación social; y sus entornos ecológicos son la familia, la escuela, el barrio, el vecindario, las instituciones,...

De tal manera que cuando nos referimos a infancia en situación de riesgo social no solamente nos referimos al maltrato dentro de la familia, sino a una situación mucho más amplia, que reúne todas aquellas circunstancias sociales carenciales para el correcto desarrollo de la infancia. (Balsells, 1997).

¿Y cuales son esas situaciones carenciales, alejadas de las causas familiares? Todas aquellas que se generan en los diferentes agentes de socialización de la infancia como por ejemplo el maltrato entre iguales, el builling, el maltrato en el deporte, a través del consumo, del urbanismo y diseño de las ciudades..situaciones generadas en el seno de las sociedades del bienestar y que han sido "bautizadas" como las nuevas formas de maltrato infantil: "Se trata de circunstancias alejadas de los "habituales malos tratos" pero que día a día van cobrando más significación, principalmente por sus repercusiones en sectores de población aparentemente alejados del maltrato infantil" (Petrus, 1997, 26).

\section{3. ¿Como detectar a la nueva infancia en riesgo social?}

Atendiendo a una reducción de los problemas de la infancia a los maltratos en la familia, el análisis de perfiles individuales de situaciones de riesgo para la infancia ha estado marcada por el estudio de indicadores que hacen prever la existencia de situaciones de máxima desprotección de los niños; es decir, por el análisis y la descripción de situaciones de maltratos y de negligencia. A través de indicadores, físicos y comportamentales en los niños y conductuales en los padres se pueden identificar situaciones de maltratos o de emergencia de esta situación. En base a la existencia de estos indicadores se inician los procesos de intervención individualizados, de prevención secundaria o terciaria, y en base a los factores de riesgo relacionados con la emergencia de los maltratos se identifican poblaciones de riesgo para poder hacer una prevención primaria. Este es el esquema usado en la intervención con la infancia maltratada en el seno familiar y autores como Gracia y Musitu 
(1993), Belsky (1980), De Paul (1996), Casas (1989), Arruabarrena (1996), entre otros, le han aportado rigor científico.

En esa misma línea, desde los servicios sociales de protección a la infancia, el análisis de situaciones de riesgo se hace en base a listados de indicadores de riesgo social que recogen los anteriores y, en ocasiones, son completados con otros relacionados con la emergencia de situaciones de inadaptación social. La Generalitat de Catalunya, la Diputación de Zaragoza o autores como Inglés (1993), Cusó (1995) o Panchón (1995) trabajan en este sentido.

La detección operativizada desde esta forma es perfectamente válida cuando se quiere centrar la protección de la infancia ante situaciones de maltrato infantil familiar en cualquiera de sus formas: maltrato físico, abuso sexual, negligencia, maltrato emocional o abandono.

Sin embargo, la cuestión que aquí se plantea es como formalizar un sistema de detección igualmente válido pero que abarque estas situaciones emergentes en nuestra sociedad y que se sitúan fuera de la responsabilidad paterna clásica. Es decir, encontrar un instrumento que nos permita estudiar estas situaciones contextuales que ponen en peligro el desarrollo de la infancia que se pueda adaptar a todos los contextos y escenarios ¿Cuales son las características de los entornos escolares, sociales, del grupo de iguales,... que favorecen las nuevas situaciones de riesgo? ¿Cuales son los factores y los indicadores en estos casos? ¿ ¿Cuáles son las características que nos indican que un niño o niña está sufriendo alguna nueva forma de maltrato infantil?

Por ejemplo, ¿la situación de refugiados de guerra o de inmigrantes permite que la infancia tenga la misma calidad de vida que si no hubieran tenido que abandonar su país de origen, en el que tienen sus raíces, su familia y en el que pueden hablar su idioma? O bien, ¿un niño víctima de maltrato, abuso y acoso emocional por un compañero del colegio, puede desarrollar su autoestima y seguridad emocional de igual forma que otro que no lo padezca?

Actualmente, el Instituto Infancia y mundo urbano (www.ciimu.org) está elaborando un sistema de indicadores orientados a la evaluación del bienestar de la población infantil y juvenil que permita representar y conceptuar la realidad actual de esta población.

Otra propuesta válida para el objetivo planteado es utilizar un instrumento desarrollado desarrollada en España por López, Fuertes Zurita, López Gómez de Cádiz, Sánchez Redondo y Merino (1995), en el Programa de mejora del Sistema de atención social a la infancia del Ministerio de Asuntos Sociales: la teoría de las necesidades de la infancia se basa en una taxonomía de necesidades fundamentada en las diferentes formulaciones de los derechos de los niños y las niñas. Esta nueva forma de análisis de situaciones que implican riesgo para el desarrollo integral permitirá operativizar nuevos indicadores.

De hecho, la utilización de la Convención de los Derechos de la Infancia como punto de referencia para realizar evaluaciones de este tipo, a través de la Taxonomía de las Necesidades Básicas, permite trasladar el criterio político y social hasta un criterio técnico y científico bajo la bandera de la universalidad. Sin embargo, es innegable la dificultad que entraña medir el grado de riesgo para la infancia de una situación, ya que es una acción impregnada de componentes culturales y éticos. La importancia de este hecho radica en las decisiones posteriores que afectarán a la vida de estos niños y niñas, ya que en función de la gravedad de su situación se determinará la acción de protección más adecuada y ésta puede llegar a ser su separación del núcleo familiar. ¿Cómo neutralizar los componentes culturales, la subjetividad del evaluador, o las diferencias establecidas desde las tradiciones? Sin duda este es un interrogante todavía sin respuesta que queda pendiente en la propia definición de la infancia en situación de riesgo social, y aunque el utilizar como referencia la Convención de los Derechos de la Infancia es de por si un gran adelanto, todavía queda mucho para llegar un aceptable grado de intersubjetividad.

Desde este Programa se describen tres grandes bloques de necesidades: las de carácter físico biológico, las de carácter cognitivo y las necesidades emocionales y sociales. Esta taxonomía de necesidades infantiles permite analizar aquellas situaciones en que, si bien no se da maltrato infantil, si que representan una situación de necesidades no cubiertas. Este planteamiento permite la mejora del 
bienestar de la infancia de forma más amplia que desde la teoría de las carencias, y también permite identificar no solo las situaciones familiares, sino también aquellas del entorno ecológico de los niños y niñas que pueden poner en riesgo su desarrollo, hasta dar paso a situaciones de desadaptación.

En definitiva, tiene en cuenta una perspectiva ecológica y interactiva, que valora todos los entornos sociales del niño, así como su interacción, y puede servir al objetivo planteado en este texto. El siguiente cuadro refleja todas las necesidades básicas, así como las situaciones correspondientes consideradas de riesgo:

\begin{tabular}{|c|c|}
\hline NECESIDAD & RIESGO \\
\hline \multicolumn{2}{|l|}{ a) Necesidades fisico-biológicas } \\
\hline - Alimentación & Desnutrición, déficit, no apropiada a la edad,. \\
\hline - Temperatura & $\begin{array}{l}\text { Frío en la vivienda, humedad, falta de vestido } y \\
\text { calzado }\end{array}$ \\
\hline - Higiene & Suciedad, parásitos,... \\
\hline - Sueño & Insuficiente, lugar inadecuado, ruido ambiental. \\
\hline - Actividad física: ejercicio y juego & $\begin{array}{l}\text { Introvilidad corporal, ausencia de juegos y de espacio, } \\
\text { inactividad }\end{array}$ \\
\hline - Protección de riscos reales & $\begin{array}{l}\text { Accidentes domésticos, castigos físicos, agresiones, } \\
\text { accidentes de circulación,.. }\end{array}$ \\
\hline - Salud & $\begin{array}{l}\text { Falta de control, provocación de sintomas, no } \\
\text { vacunación,.. }\end{array}$ \\
\hline \multicolumn{2}{|l|}{ b) Necesidades cognitivas } \\
\hline - Estimulación sensorial & $\begin{array}{l}\text { Falta de estimulación lingüistica, privación o pobreza } \\
\text { sensorial, retraso en el desarrollo no orgánico,.. }\end{array}$ \\
\hline - Exploración física i social & No tener apoyo en la exploración, entomo pobre,... \\
\hline - Comprensión de la realidad física y social & $\begin{array}{l}\text { No escuchar, no responder, mentir, visión pesimista, } \\
\text { anomia o valores antisociales,... }\end{array}$ \\
\hline \multicolumn{2}{|l|}{ c) Necesidades e mocionales i sociales } \\
\hline - Seguridad emocional & $\begin{array}{l}\text { Rechazo, ausencia, no accesibles, no responder, no } \\
\text { percibir,.. }\end{array}$ \\
\hline - Red de relaciones sociales & $\begin{array}{l}\text { Aislamiento social, imposibilidad de contactar con } \\
\text { amigos, compañeros de riesgo,.. }\end{array}$ \\
\hline - Participación y autonomía progresiva & No ser escuchar, dependencia, ... \\
\hline - Curiosidad, imitación y contacto sexual & $\begin{array}{l}\text { No escuchar, no responder, engañar, castigar } \\
\text { manifestaciones infantiles, abuso sexual,.. }\end{array}$ \\
\hline - Protección de riesgos imaginarios & $\begin{array}{l}\text { No escuchar, no responder, no tranquilizar, violencia } \\
\text { verbal, amenazas, perdida de control, ... }\end{array}$ \\
\hline - Interacción lúdica & $\begin{array}{l}\text { No disponibilidad de tiempo, no accesibilidad, } \\
\text { ausencia de iguales,... }\end{array}$ \\
\hline
\end{tabular}

Cuadro I: Taxonomía de las Necesidades Básicas: (Adaptación: López, y otros, 1995)

\section{4.- Los nuevos colectivos en situación de riesgo social}

Partiendo de la base que son estas necesidades las que cualquier menor debe tener cubiertas para desarrollarse de forma integral, podemos empezar a operativizar y a definir situaciones que están emergiendo en las sociedades más desarrolladas, y que plantean nuevas situaciones de riesgo social. Se trata de situaciones en las que la infancia puede tener una familia que cumpla sus funciones parentales, pero que tiene algún otro entorno ecológico, que forma parte de su socialización secundaria, y que por acción u omisión no cubre sus necesidades. El siguiente cuadro resume dicha relación a través de algunos ejemplos de nuevos colectivos en situación de riesgo social. 


\begin{tabular}{|c|c|c|}
\hline $\begin{array}{c}\text { Entorno ecológico de la } \\
\text { infancia }\end{array}$ & $\begin{array}{c}\text { Grupo de necesidades no } \\
\text { cubiertas }\end{array}$ & $\begin{array}{c}\text { Fenómenos que favorecen } \\
\text { la aparición de colectivos } \\
\text { en situación de riesgo } \\
\text { social }\end{array}$ \\
\hline Familia & $\begin{array}{l}\text { Necesidades fisicas, biológicas, } \\
\text { cognitivas, sexuales, sociales y } \\
\text { afectivas. }\end{array}$ & Maltrato infantil familiar \\
\hline Grupo de iguales & $\begin{array}{l}\text { Necesidades fisicas, biológicas, } \\
\text { cognitivas, sexuales, sociales y } \\
\text { afectivas. }\end{array}$ & Maltrato entre iguales \\
\hline Escuela & Necesidades sociales y cognitivas & Violencia escolar \\
\hline Sociedad & $\begin{array}{c}\text { Necesidades sociales } \\
\text { Necesidades cognitivas }\end{array}$ & $\begin{array}{c}\text { Movimientos migratorios } \\
\text { Mass media }\end{array}$ \\
\hline
\end{tabular}

Cuadro II: Nuevos grupos de infancia en situación de riesgo social

\section{1.- El maltrato entre iguales}

El maltrato entre iguales ha sido definido como "un comportamiento prolongado de insulto verbal, rechazo social intimidación psicológica y/o agresividad física de unos niños hacia otros que se convierten, de esta forma, en víctimas de sus compañeros" (Olweus, 1993).

Por lo tanto, se trata de una forma de maltrato activo que se da dentro del microsistema y la microcultura propia del grupo de iguales, en la que se producen daños físicos y psicológicos a través de un uso indiscriminado y deshonesto del poder.

Este fenómeno, que se suele dar en el marco escolar, pero que también se puede generar en otros contextos (las pandillas de la calle, en los recursos de tiempo libre,...) constituye una clara situación de riesgo social fundamentalmente para las víctimas, pero también para los agresores y los observadores. Todos ellos están haciendo un aprendizaje social y construyendo un esquema de socialización, en el que los límites del poder están en la agresividad y la prepotencia, alterando cualquier esquema normalizado de dominio-sumisión.

Las necesidades básicas de la infancia que padece esta situación quedan alteradas y son vulneradas en mayor o en menor medida, según la gravedad del maltrato; efectivamente, si existen agresiones físicas del tipo pegarse entre compañeros o agredir con algún tipo de instrumento (navajas, palos,...) nos encontramos ante una situación en que la protección de riesgos reales está descubierta ya que la integridad física de las víctimas no está garantizada.

La necesidad básica de comprensión de la realidad física y social consistente en la transmisión y asimilación de las actitudes y los valores prosociales también se ve claramente alterada: los códigos morales que son aprendidos en un contexto de maltrato entre iguales sitúan en una clara situación de riesgo social a víctimas, agresores y observadores del fenómeno. Los efectos de una socialización enmarcada en estos parámetros son claros factores de riesgo para la desadaptación social.

Cuando se da cualquier forma de maltrato entre iguales, ya sea el maltrato físico, el psicológico (insultos, amenazas, desprecios, propuestas de tipo sexual, ignorar, ofender,... ) o ambos, son las necesidades de seguridad emocional y de tener una red de relaciones sociales las que siempre quedan descubiertas. La creación de la autoestima, tan relacionada con vivir y crecer en un clima de aceptación, se ve minada:

" Si la microcultura de los iguales incluye claves simbólicas de dominio y sumisión interpersonal, y la realidad cotidiana de la relación incluye el desprecio, la falta de consideración y, finalmente, los malos tratos, el grupo de iguales pasa a ser un espejo en el cual ir observando el crecimiento de uno mismo/a, a convertirse en un espejo roto en mil pedazos, en el cual se aparece desfigurado y fragmentado en la identidad propia y dañado en la 
Finalmente, para el desarrollo integral de la infancia el establecimiento de relaciones de amistad y de compañerismo es una pieza clave que permite la paulatina creación de actitudes positivas hacia los individuos en pro a la sociabilidad de los mismos. El peligro ante estas situaciones de maltrato entre iguales es que se integren patrones $y$ actitudes que van a encaminar hacia una vida social distorsionada.

\section{2.- La violencia escolar}

La violencia escolar es un fenómeno que ha ido surgiendo en los países occidentales más avanzados y que cada vez preocupa más. El estudio de sus causas, consecuencias y de las posibles soluciones está ocupando un lugar importante en la investigación y la publicación científica.; este fenómeno ha sido denunciado por los medios de comunicación cuando se ha llegado a casos extremos y llamativos, pero sin embargo se puede dar en diferentes formas y grados, y todas ellas perturban el buen funcionamiento educativo de la institución escolar.

La violencia escolar reúne bajo su epígrafe una serie de conductas diversas y prolongadas en el tiempo que dificultan y alteran la convivencia de la comunidad educativa, siendo provocado por un alumno que es apoyado por el grupo, y mantenido por el resto, los cuales son pasivos o ignorantes de los hechos ((Díaz-Aguado, 1999). Adaptando la clasificación de (Fernández, 1998) podríamos decir que existen diferentes formas de violencia escolar: los actos disruptivos son aquellos que se suelen dar dentro del aula y que generan un clima y un ambiente poco propicio para cumplir con las funciones educativas (llegar tarde, no hacer los deberes, hablar mientras el profesor explica, malas posturas, fumar, comer, hablar o enviar mensajes con el móvil, estar despistado,...). Se trata de actos del alumnado muy relacionados con la indisciplina escolar que generan un fuerte malestar docente; esta forma de violencia escolar es la menos grave pero sin duda la que más se da en nuestro entorno inmediato. Otra tipología es la violencia propiamente dicha, que se puede dirigir a profesores (insultos, dañar coches, agresiones, burlas,...), a alumnos (seria el maltrato entre iguales), a bienes (robar material escolar, romper instalaciones, quemar material, inutilizar cerraduras,) o a la institución (absentismo, faltas injustificadas, rechazo escolar,...). Esta violencia puede ser física, verbal, psicológica o indirecta.

Ayerbe (2002) plantea una panorámica de algunas de las explicaciones sociales y escolares de la violencia escolar: prolongación de la escolaridad obligatoria, la integración laboral, la normatividad y justicia, la segregación escolar y las vivencias de los alumnos.

En cualquiera de sus formas y en cualquiera de sus diferentes intensidades, la violencia escolar genera un grado de desprotección de la infancia que se concreta fundamentalmente en la localización de factores de riesgo referentes a sus necesidades cognitivas y sociales.

La institución escolar tiene una clara función relacionada con el desarrollo de las habilidades y capacidades cognitivas de la infancia y la adolescencia, con la finalidad de formar sujetos que se puedan integrar en la sociedad de forma positiva. Esto conlleva un trabajo de transmisión de valores de tolerancia y de respeto hacia los otros, independientemente de su raza, nacionalidad o sexo, así como de respeto a los bienes materiales propios y comunes. Sin embargo, cuando la institución escolar se convierte en el escenario de la violencia escolar, se esta dando un claro riesgo que la construcción social del individuo sea en base al racismo, al dogmatismo, a la ley del silencio y/o a la ley del más fuerte. Los valores antisociales que existen detrás de cualquier forma de violencia pueden convertirse en los referentes de los alumnos que los están viendo, asimilando y sufriendo día tras día. Todo ello sin olvidar que también conlleva un perjuicio respecto a las necesidades sociales propias de las edades escolares.

¿Un niño que observa de forma constante como sus compañeros insultan a los profesores tiene las mismas posibilidades de aprendizaje social que otro que vive en un clima positivo de convivencia 
escolar? ¿Una niña que protagoniza capítulos habituales de insultos y burlas machistas, está recibiendo una correcta socialización secundaria? En definitiva, ¿es posible afirmar que los actos disruptivos, así como la violencia escolar más grave son factores de riesgo de la desadaptación social? Parece ser que la respuesta es afirmativa, poniendo sobre la mesa la necesidad de hacer actuaciones multidimensionales en situaciones de riesgo social que se dan fuera de los entornos familiares.

\section{3.- Movimientos migratorios}

La llegada a los países europeos de ciudadanos de países en vías de desarrollo que no se pueden ganar la vida en su país de origen es uno de los fenómenos sociales que se está viviendo con más fuerza y más dificultades en la actualidad: políticos, legisladores, responsables educativos, actores sociales,... todos ellos están en vías de analizar la situación y sus posibles soluciones.

En relación a la infancia que vive los movimientos migratorios nos encontramos con casuísticas diferentes; por un lado está el/la hijo/a del emigrante que forma parte de una familia que no se separa y se traslada toda junta a un país desarrollado; también encontramos niños/as que ven como su familia se tiene que separar porque el padre o la madre emigran a otro país; o también el caso de adolescentes que emigran solos a un país europeo con la esperanza de encontrar trabajo. En todos estos casos, así como en el resto de situaciones producidas por la emigración, nos encontramos ante una problemática social que genera una situación de riesgo para la infancia que la vive.

Cualquier menor que ha tenido que dejar su país de origen y llegar a otro donde la lengua, las costumbres, los usos y los valores nada tienen que ver con los propios, se encuentra en una situación de desventaja social en relación a las posibilidades de adaptarse de forma ajustada. La vulneración de sus derechos relacionados con las necesidades sociales es evidente cuando se piensa en las posibilidades de establecer una red de relaciones sociales: el plano de igualdad es difícil de encontrar en el grupo de iguales cuando no compartes lengua, soportar actitudes racistas y xenófobas por el simple hecho de no ser autóctono o no poder celebrar las fiestas religiosas propias, son algunos ejemplos del aislamiento social que pueden vivir estos/as niños/as.

Sin embargo, la vertiginosa evolución de la sociedad en la que vivimos, hace que los movimientos migratorias vayan provocando nuevas situaciones a cada momento. La emigración es una realidad cambiante en si misma: ello nos obliga a estar atentos a las nuevas situaciones de riesgo que se puedan generar.

Un ejemplo de ello es el colectivo de adolescentes emigrantes que están desamparados; se calcula que en la ciudad de Barcelona existen 300 menores de edad magrebíes que viven en la calle. Son chicos/as que emigran sin su familia a nuestro país con la esperanza de encontrar trabajo, pero lo hacen sin documentación, sin tener la edad necesaria para incorporarse al mundo laboral y, por su puesto, sin calificación profesional. Ello ha generado un colectivo en riesgo social caracterizado por la movilidad geográfica, un desarraigo social y familiar, sin identificación y que cometen pequeños delitos para sobrevivir en el país que les acoge.

La sociedad no ha estado preparada para dar respuesta al cubrimiento de las necesidades básicas de este nuevo colectivo en riesgo, ya que ha aparecido de forma muy reciente. Así, estos menores, que en el mejor de los casos eran declarados desamparados para ser tutelados y protegidos por la Administración, en la mayoría de los casos rechazan esta tutela huyendo de centros de acogida que están pensados y organizados para un tipo de menores de características y expectativas muy alejadas a las suyas. Definitivamente, se han tenido que crear nuevos recursos para atender a este nuevo colectivo.

\section{4.- Los mass media}

"los mass media aparecen como centros difusores de cultura, haciendo tambalear los cimientos del centro difusor de cultura por excelencia, la escuela. Ésta ha perdido el monopolio de la 
transmisión cultural y sus alumnos se nutren ahora en los mass media, ya que la escuela se ha visto imposibilitada para integrar las formas culturales contemporáneas a las que, por otra parte, ya está adheridos los alumnos" (Salinas, 1992:253)

En esta cita se resalta la importancia que en la actualidad tienen los medios de comunicación como transmisor de las concepciones de los grupos dominantes de la sociedad, y más concretamente la televisión. La televisión ha impuesto nuevas pautas de vida cotidiana, hasta el punto que es un miembro más de la familia, y en función de la que se decide los horarios domésticos.

Con relación a la infancia en riesgo social, se ha estudiado la televisión como un medio de comunicación que favorece determinados aprendizajes que pueden convertirse, si su uso no es el correcto, en un entorno socializador de riesgo. El consumismo y la violencia que transmiten son dos de los contenidos más estudiados.

El consumismo, a través de la publicidad que relaciona la adquisición de determinados productos con el éxito social y personal, favorece los sentimientos de frustración de determinados sectores de la población con poco poder adquisitivo.

\footnotetext{
"Continuamente el discurso del consumo apela a las motivaciones psicológicas de las personas para activarlas, potenciarlas y asegurar que con la adquisición de objetos se verán satisfechas: la seguridad, el poder, el sexo, la autoafirmación, la aprobación de los demás,... Sin embargo, la insatisfacción es permanente puesto que el número de objetos a poseer nunca deja de crecer y aumentar, y es imposible de alcanzar" (Pérez, 1992,123)
}

La violencia en la televisión y el estudio de las consecuencias en la agresividad y las conductas violentas de los niños también es frecuente y llega a conclusiones que relacionan una y otra variable en función de otras características. Un reciente estudio de la Universidad de Michigan, desarrollado durante 15 años, ha llegado a la conclusión que los/as niños/as que ven programas violentos en televisión están más predispuestos a protagonizar actitudes y respuestas agresivas cuando son adultos. A su vez, autores como por ejemplo Petrus (2001) han denunciado la capacidad de modelling de los mass media como una de las causas de que los escolares adopten como propios, patrones de conducta violentos y agresivos. Resulta contradictorio que la sociedad actual, con el uso y abuso de la televisión, pretenda cubrir la necesidad básica de comprensión social; la contradicción radica en el mismo contenido de los programas televisivos: actitudes violentas, agresivas, racistas, sexistas,. Frente a los contenidos y los valores que esa misma sociedad dice y reclama que se transmitan a través de la familia, la escuela o los centros educativos no formales.

En definitiva, la aparición de nuevas poblaciones en riesgo de exclusión, desadaptación o inadaptación social, tiene mucho que ver con las características y los medios de socialización secundaria que se van generando en las sociedades avanzadas; prevenir sus consecuencias y la consolidación de las mismas, requiere un esfuerzo multidimensional e interdisciplinar, en actuaciones planteadas desde el modelo ecológico y la acción por redes, dado que todos los entornos son la causa y, a su vez, la solución. La complejidad d del fenómeno requiere actuaciones globales que abarquen medidas políticas, legales, escolares, familiares, Tal y como se plantea en la declaración de Copenhague de 1995 sobre el desarrollo social, la sociedad debe ser sensible al desarrollo y el bienestar social de todos los sectores sociales, y de forma particular de la infancia que vive situaciones vulnerables.

\section{Bibliografía}

AYERBE, P. (2000) Convivencia y violencia en la ESO: aspectos didácticos organizativos, en Lorenzo, M.; Garcia, M.D. ; Torres, J.A.; Ortega, J.A.; Debón, S. y Notoria, A. (eds) Las organizaciones educativas en la sociedad neoliberal. Granada, Grupo editorial universitario, 339-368.

BALSELLS, A. (1997) Maltractament infantil i educació familiar. Lleida, Universitat de Lleida.

BELSKY, J. (1980) Child maltreatment. An ecological integration. American Psychologist, 35, 320- 
335.

CUSÓ, M. (1995), Infancia en riesgo e infancia maltratada. Comunicación y Lenguaje, 27, 87-97

DE PAUL OCHOTORENA, J. y ARRUABARRENA, M.I (1996), Manual de protección infantil. Barcelona, Masson.

DÍAZ-AGUADO, M.J. (1999). Programas de educación para la tolerancia y prevención de la violencia en los jóvenes. Volumen 1. Fundamentación psicopedagógica. Madrid, Ministerio de trabajo $\mathrm{y}$ asuntos sociales.

FERNANDEZ, I. (1998) Prevención de la violencia y resolución de conflictos. Madrid, Narcea.

GRACIA, E. \& MUSITU, G. (1993) El maltrato infantil. Un análisis ecológico de los factores de riesgo. Madrid, Centro de Publicaciones del Ministerio de Asuntos Sociales.

GOMEZ-GRANELL, C.; GARCIA-MILA, M.; RIPOL-MILLET, A. y PANCHÓN, C. (cood) (2002) Informe 2002. La infància i les famílies als inicis dels segle XXI.. Barcelona, Institut d'infància i món urbà. Observatori de la infància i la família.

GUASCH, M. y PONCE, C. (2002) ¿Qué significa intervenir educativamente en desadaptación social? Barcelona, ICE Universidad de Barcelona.

INGLÉS, A. (1993), Els maltractaments infantils a Catalunya. Dossier, 8, 9-12.

LOPEZ, F.; LOPEZ, B.; FUERTES, J. SANCHEZ, J.M. y MERINO, J. (1995): Necesidades de la infancia y protección infantil. Madrid, Ministerio de Asuntos Sociales.

OLWEUS, D. (1993) Bullying at scholl. What we know and what we can do. Oxford. U.K. , Blackwell.

ORTEGA, R. y colaboradores (1998) La convivencia escolar: que es y como abordarla. Sevilla, Conserjería de Educación y Ciencia. Junta de Andalucía.

PANCHÓN, C. (1995), Intervención con familias en situaciones de alto riesgo social. Comunicación, lenguaje y educación. 27. 61-74.

PÉREZ, J. M. et al. (1992): La seducción de la opulencia. Barcelona: Paidós

PETRUS, A. (1997) Nuevas formas de maltrato infantil en BALSELLS, A., Maltractament infantil $i$ educació familiar. Lleida, Universitat de Lleida., 9-45

PETRUS, A. (2001). Cultura de la violencia y educación secundaria. Revista española de educación comparada. 7.23-51

SALINAS, J. (1992): Mass-media y Educación Social. en COLOM, A.J., Modelos de intervención socioeducativa. Narcea. 252-276.

(C) Ediciones Universidad de Salamanca 